sion", Vol. 1 (1953).

(3) M. OTsukA : This Journal, 2, No.5, 276 (1960), (in Japanese).
(4) R. T. Ackroyd, J. D. McCullen : Proc. 2nd U. N. Intern. Conf. Peaceful Uses of Atomic Energy, 12, 38, P/16 (1958).

\title{
研究論文
}

\section{An Analysis of Dynamic Characteristics of Plutonium-and/or Thorium-recycled Reactor, (I)}

Reactor kinetic equations

\author{
プルトニウム,トリウムリサイクル系に拈ける \\ 原子炉動特性の解析, (I) \\ 原子炉動特性方程式
}

By Kunihiko UEMATSU

\begin{abstract}
Starting from the neutron balance equation, the reactor kinetic equations which are generally applicable to a plutonium- and/or thorium-recycled reactor are derived. The use of the newly defined effective fraction of delayed neutrons, $\beta_{\text {eff }}$, with these reactor kinetic equations simplifies the analysis of the dynamic behavior of a plutonium- and/or thorium-recycled reactor.
\end{abstract}

\section{INTRODUCTION}

It is important to clarify the transient behavior of the reactor from the view point of both reactor safety and control- especially for the plutonium recycle case, which usually has a positive overall temperature coefficient of reactivity. The usual reactor kinetic equations applicable to the monospecied fissile material are well known, however, the reactor kinetic equations which are applicable to the plutonium and/or thorium recycle cases have not been well defined. These kinetic equations for plutonium and/or thorium recycle are very important in view of a steadily increasing possibility of plutonium and/or thorium recycle in power reactors. The author derived the kinetic equations applicable to both recycle cases starting from the neutron balance equation, and defined the effective fraction of delayed neutrons, $\beta_{\text {esf }}$, for the case of plutonium and/or thorium recycle.

\section{Derivation of Basic Equations}

The neutron flux of a homogeneous bare reactor is given by the neutron balance equation

$$
\begin{aligned}
\frac{1}{v} \frac{\partial \phi(r, t)}{\partial t}= & \operatorname{div} D \operatorname{grad} \phi(r, t) \\
& -\Sigma_{a}^{F} \phi(r, t)+S(r, t),
\end{aligned}
$$

where

$$
\begin{aligned}
\Sigma_{a}^{F}= & \Sigma_{a}^{25}+\Sigma_{a}^{28}+\sum_{a}^{49}+\Sigma_{a}^{40} \\
& +\sum_{a}^{41}+\Sigma_{a}^{42}+\Sigma_{a}^{23}+\Sigma_{a}^{03} .
\end{aligned}
$$

* 植松邦彦。Dept. Nucl. Engng., Kyoto Univ. (京 都大学 工学部原子核工学教室)

** The superfixes, 25,28 , etc. of equations through this paper set such that the first digit denotes to the last digit of the atomic number and the second digit denotes to the last digit of the atomic weight. Therefore, 25 means ${ }^{235} \mathrm{U}_{92}$. 
The source term is

$$
\begin{aligned}
S(r, t)= & \left(1-\sum_{i} \beta_{i} \gamma_{j}\right) \varepsilon_{25} \eta_{25} P_{25} \sum_{a}^{25} \phi(r, t) \\
& +\left(1-\sum_{j} \beta_{j} \gamma_{j}\right) \varepsilon_{49} \eta_{49} P_{49} \sum_{a}^{48} \phi(r, t) \\
& +\left(1-\sum_{k} \beta_{k} \gamma_{k}\right) \varepsilon_{41} \eta_{41} P_{41} \sum_{a}^{41} \phi(r, t) \\
& +\left(1-\sum_{m} \beta_{m} \gamma_{m}\right) \varepsilon_{23} \eta_{23} P_{23} \sum_{a}^{23} \phi(r, t) \\
& +\sum_{i} P_{i} \lambda_{i} C_{i}(r, t) \\
& +\sum_{j} P_{j} \lambda, C_{j}(r, t) \\
& +\sum_{k} P_{k} \lambda_{k} C_{k}(r, t) \\
& +\sum_{m} P_{m} \lambda_{m} C_{m}(r, t)+S_{0}(r, t) \quad(3) \\
& i, j, k, m=1, \cdots \cdots, 6,6
\end{aligned}
$$

where

$$
\begin{aligned}
& \eta_{25}=\frac{\nu^{25} \sum_{f}^{25}}{\sum_{0}^{25}} \\
& \eta_{49}=\frac{\nu^{49} \sum_{\rho}^{49}}{\sum_{a}^{49}} \\
& \eta_{41}=\frac{y^{41} \sum_{f}^{41}}{\sum_{a}^{1 !}} \\
& \eta_{23}=\frac{\nu^{23} \sum_{j}^{23}}{\sum_{a}^{23}}
\end{aligned}
$$

$S_{0}(r, t):$ Extraneous neutron source.

The rate of formation of delayed neutron emitters is given by

$$
\frac{d C_{t}(r, t)}{d t}=\varepsilon \eta_{25} \beta_{1} \sum_{a}^{25} \phi(r, t)-\lambda_{i} C_{t}(r, t)
$$

$$
\begin{aligned}
& \frac{d C_{j}(r, t)}{d t}=\varepsilon \eta_{49} \beta_{j} \Sigma_{a}^{49} \phi(r, t)-\lambda, C_{j}(r, t) \\
& \frac{d C_{k}(r, t)}{d t}=\varepsilon \eta_{41} \beta_{k} \Sigma_{a}^{41} \phi(r, t)-\lambda_{k} C_{k}(r, t) . \\
& \frac{d C_{m}(r, t)}{d t}=\varepsilon \eta_{23} \beta_{m} \Sigma_{a}^{23} \phi(r, t)-\lambda_{m} C_{m}(r, t)(5) \\
& i, j, k, m=1, \cdots \cdots \cdots \cdots, 6 .
\end{aligned}
$$

Because the first mode of the neutron flux is nearly always predominant, it can be assumed to be separable into time- and space-dependent functions :

$$
\phi(r, t)=\phi(r) \phi(t) .
$$

It is also assumed that the extraneous source and the delayed neutron precursor have space dependence on the flux :

$$
\begin{aligned}
& S_{0}(r, t)=\phi(r) S_{0}(t) \\
& C_{i, j, \boldsymbol{k}, \boldsymbol{m}}(r, t)=\phi(r) C_{\boldsymbol{t}, y, \boldsymbol{k}, \boldsymbol{m}}(t),
\end{aligned}
$$

and that the space-dependent flux $\phi(r)$ satisfies the steady-state equation of the critical reactor :

$$
\begin{aligned}
& \operatorname{div} D \operatorname{grad} \phi(r)+\varepsilon \eta_{25} P \sum_{a}^{25} \phi(r)+\varepsilon \eta_{49} P \sum_{a}^{49} \phi(r) \\
& +\varepsilon \eta_{41} P \sum_{a}^{41} \phi(r)+\varepsilon \eta_{23} P \sum_{a}^{33} \phi(r)-\sum_{a}^{P} \phi(r)=0
\end{aligned}
$$

or

$$
\nabla^{2} \phi(r)+B^{2} \phi(r)=0 .
$$

Using the effective neutron lifetime

$$
l=\frac{1}{v \sum_{\boldsymbol{a}}^{P}\left(1+L^{2} B^{2}\right)},
$$

$L^{2}=D / \Sigma_{a}^{P}$ and Eqs. (1), (2), (6), (7), (8), (9) and $(10)$, the neutron density equation becomes,

$$
\begin{aligned}
\frac{d n(t)}{d t}= & -\frac{n(t)}{l} \\
& +v \varepsilon \eta_{25} P\left(1-\sum_{i} \beta_{i} \gamma_{t}\right) \sum_{a}^{25} n(t) \\
& +v \varepsilon \eta_{49} P\left(1-\sum_{j} \beta_{j} \gamma_{j}\right) \sum_{a}^{49} n(t) \\
& +v \varepsilon \eta_{41} P\left(1-\sum_{k} \beta_{k} \gamma_{k}\right) \sum_{a}^{41} n(t) \\
& +v \varepsilon \eta_{23} P\left(1-\sum_{m} \beta_{m} \gamma_{m}\right) \sum_{a}^{23} n(t) \\
& +\sum_{i} P_{t} \lambda_{i} C_{t}(t) \\
& +\sum_{j} P_{j} \lambda_{j} C_{j}(t) \\
& +\sum_{k} P_{k} \lambda_{k} C_{k}(t) \\
& +\sum_{m} P_{m} \lambda_{m} C_{m}(t)+S_{0}(t) \\
& \quad i, j, k, m=1, \cdots \cdots \cdots \cdots, 6 .
\end{aligned}
$$


Defining the new effective multiplication factor as

$$
k_{e f f}=\frac{\varepsilon \bar{\eta} P}{1+L^{2} B^{2}}
$$

where

$$
\bar{\eta}=\frac{\nu^{25} \sum_{f}^{28}+\nu^{48} \sum_{j}^{48}+\nu^{41} \sum_{f}^{4 i}+\nu^{23} \sum_{f}^{23}}{\Sigma_{a}^{F}},
$$

and combining Eqs. (11), (12) and (13),

$$
\begin{aligned}
\frac{d n(t)}{d t}= & -\frac{n(t)}{l}+\frac{k_{e f}\left(1-\sum_{i} \beta_{i} \gamma_{t}\right)}{l} n(t) \frac{\eta_{25}}{\bar{\eta}} \frac{\sum_{a}^{2 s}}{\sum_{a}^{F}} \\
& +\frac{k_{e f}\left(1-\sum_{j} \beta_{j} \gamma_{j}\right)}{l} n(t) \frac{\eta_{49}}{\bar{\eta}} \frac{\sum_{a}^{49}}{\sum_{a}^{F}} \\
& +\frac{k_{e f f}\left(1-\sum_{k} \beta_{k} \gamma_{k}\right)}{l} n(t) \frac{\eta_{41}}{\bar{\eta}} \frac{\sum_{a}^{41}}{\sum_{a}^{F}} \\
& +\frac{k_{e f}\left(1-\sum_{m} \beta_{m} \gamma_{m}\right)}{l} n(t) \frac{\eta_{23}}{\bar{\eta}} \frac{\sum_{a}^{23}}{\sum_{a}^{F}} \\
& +\sum_{i} P_{i} \lambda_{t} C_{t}(t)+\sum_{j} P_{j} \lambda_{j} C_{j}(t)+\sum_{k} P_{k} \lambda_{k} C_{k}(t) \\
& +\sum_{m} P_{m} \lambda_{m} C_{m}(t)+S_{0}(t) \\
& i, j, k, m=1, \cdots \cdots \cdots, .
\end{aligned}
$$

From Eqs. (5), (6), (7) and (12),

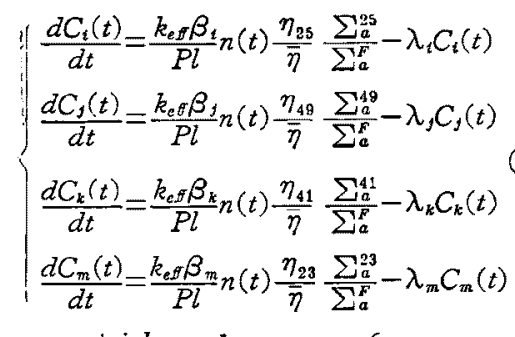

$$
\begin{aligned}
& i, j, k, m=1, \cdots \cdots \cdots \cdots, 6 \text {. }
\end{aligned}
$$

Ordinarily we may take

$$
\left\{\begin{array}{l}
P_{t}=P_{j}=P_{k}=P_{m}=1 \\
\gamma_{t}=\gamma_{\jmath}=\gamma_{k}=\gamma_{m}=1 \\
S_{0}(t)=0 .
\end{array}\right.
$$

Rewriting the variables of Eqs. (14) and (15),

$$
\left\{\begin{array}{cc}
\sum_{i} \beta_{j} \gamma_{i}=\beta_{I} & \sum_{i} \beta_{i} \lambda_{i}=\lambda_{I} \beta_{I} \\
\sum_{j} \beta_{j} \gamma_{j}=\beta_{J} & \sum_{j} \beta_{j} \lambda_{j}=\lambda_{J} \beta_{J} \\
\sum_{k} \beta_{k} \gamma_{k}=\beta_{K} & \sum_{k} \beta_{k} \lambda_{k}=\lambda_{R} \beta_{K} \\
\sum_{m} \beta_{m} \gamma_{m}=\beta_{M} & \sum_{m} \beta_{m} \lambda_{m}=\lambda_{\mu} \beta_{M} \\
k_{c f f}=1+k_{t z .} .
\end{array}\right.
$$

$$
\begin{aligned}
& \frac{d n(t)}{d t}=-\frac{n(t)}{l} \\
& +\frac{\left(1+k_{e x}\right)\left(1-\beta_{t}\right)}{l} n(t) \frac{\eta_{25}}{\bar{\eta}} \frac{\sum_{a}^{25}}{\sum_{a}^{F}} \\
& +\frac{\left(1+k_{e s}\right)\left(1-\beta_{J}\right)}{l} n(t) \frac{\eta_{49}}{\bar{\eta}} \frac{\sum_{a}^{48}}{\sum_{a}^{F}} \\
& +\frac{\left(1+k_{e x}\right)\left(1-\beta_{K}\right)}{l} n(t) \frac{\eta_{41}}{\bar{\eta}} \frac{\sum_{a}^{4 L}}{\sum_{a}^{F}} \\
& +\frac{\left(1+k_{e x}\right)\left(1-\beta_{M}\right)}{l} n(t) \frac{\eta_{23}}{\bar{\eta}} \frac{\sum_{a}^{23}}{\sum_{a}^{F}} \\
& +\sum_{i} \lambda_{i} C_{i}(t)+\sum_{j} \lambda_{j} C_{j}(t) \\
& +\sum_{k} \lambda_{k} C_{k}(t)+\sum_{m} \lambda_{m} C_{m}(t) \\
& i, j, k, m=1, \cdots \cdots \cdots \cdots, 6 \text {, } \\
& \left\{\begin{array}{l}
\frac{d C_{i}(t)}{d t}=\frac{\left(1+k_{e x}\right) \beta_{i}}{l} n(t) \frac{\eta_{25}}{\bar{\eta}} \frac{\sum_{a}^{25}}{\sum_{a}^{F}} \\
\frac{d C_{j}(t)}{d t}=\frac{\left(1+k_{e x}\right) \beta_{j}}{l} n(t) \frac{\eta_{49}}{\bar{\eta}} \frac{\sum_{a}^{4 \theta}}{\sum_{a}^{F}} \\
\frac{d C_{k}(t)}{d t}=\frac{\left(1+k_{b x}\right) \beta_{k}}{l} n(t) \frac{\eta_{41}}{\bar{\eta}} \frac{\sum_{n}^{41}}{\sum_{a}^{P}} \\
\frac{d C_{m}(t)}{d t}=\frac{\left(1+k_{c x}\right) \beta_{m}}{l} n(t) \frac{\eta_{23}}{\bar{\eta}} \frac{\sum_{a}^{23}}{\sum_{a}^{F}}
\end{array}\right. \\
& i, j, k, m=1, \cdots \cdots \cdots \cdots, 6 \text {. }
\end{aligned}
$$

As can be seen easily, Eqs. (18) and (19) can be reduced to the ordinary reactor kinetic equations neglecting plutoniums and ${ }^{233} \mathrm{U}$.

Certainly the six-group delayed neutrons for each of fissile atoms and four different fissile atoms make the reactor kinetic equations very complicated and make it hard to solve these equations at a high power of the reactor. However, we knew from the analysis of the ordinary reactor kinetic equations that the one group of delayed neutrons approximation agreed fairly well with the experimental results, besides reducing the complexity of the problems. It has been proved that the results of the analysis with the above-mentioned approximation are on the safe side.

Thus, from Eqs. (18) and (19), the reactor kinetic equations become

$$
\begin{aligned}
& \frac{d n(t)}{d t}=-\frac{n(t)}{l}+\frac{\left(1+k_{c x}\right)\left(1-\beta_{e f}\right)}{l} n(t) \\
& +\lambda_{I} C_{I}(t)+\lambda_{J} C_{J}(t)+\lambda_{K} C_{K}(t) \\
& +\lambda_{M C} C_{M}(t) \text {, } \\
& \left\{\begin{array}{l}
\frac{d C_{I}(t)}{d t}=\frac{\left(1+k_{e x}\right) \beta_{I}}{l} n(t) \frac{\eta_{25}}{\bar{\eta}} \frac{\sum_{l}^{25}}{\sum_{a}^{F}}-\lambda_{I} C_{I}(t) \\
\frac{d C_{J}(t)}{d t}=\frac{\left(1+k_{e x}\right) \beta_{J}}{l} n(t) \frac{\eta_{49}}{\bar{\eta}} \frac{\sum_{a}^{49}}{\sum_{a}^{F}}-\lambda_{J} C_{J}(t)
\end{array}\right.
\end{aligned}
$$




$$
\left\{\begin{array}{l}
\frac{d C_{K}(t)}{d t}=\frac{\left(1+k_{e x}\right) \beta_{K}}{l} n(t) \frac{\eta_{41}}{\bar{\eta}} \frac{\sum_{a}^{41}}{\sum_{a}^{F}}-\lambda_{K} C_{K}(t) \\
\frac{d C_{K}(t)}{d t}=\frac{\left(1+k_{e x}\right) \beta_{M}}{l} n(t) \frac{\eta_{23}}{\bar{\eta}} \frac{\sum_{a}^{23}}{\sum_{a}^{F}}-\lambda_{M} C_{M}(t),
\end{array}\right.
$$

where

$$
\begin{aligned}
\beta_{e f f}= & \beta_{I} \frac{\eta_{25}}{\bar{\eta}} \frac{\sum_{a}^{25}}{\sum_{a}^{F}}+\beta_{J} \frac{\eta_{49}}{\bar{\eta}} \frac{\sum_{a}^{49}}{\sum_{a}^{F}} \\
& +\beta_{K} \frac{\eta_{41}}{\bar{\eta}} \frac{\sum_{a}^{41}}{\sum_{a}^{F}}+\beta_{M} \frac{\eta_{23}}{\bar{\eta}} \frac{\sum_{n}^{23}}{\sum_{a}^{F}}
\end{aligned}
$$

Therefore, the analysis of the dynamic behavior of the plutonium-and/or thoriumrecycled reactor can be simplified with the use of the effective fraction of delayed neutrons, $\beta_{\text {ess. }}$.

\section{Constant Power Removal Model}

In the analysis of the transient behavior of the reactor, the model consisting of the constant power removal, one group delayed neutron, and the constant amount of delayed neutron precursor was used to avoid too much complication of the analysis. This model does not hold when the temperature increases significantly and is an unrealistic representation of actual reactor kinetics long after the introduction of a change in the reactivity.

At least, the constant-power-removal model is good for a time short in comparison with the thermal relaxation time, and shows good agreement with experimental results.

The neutron density equation for one group delayed neutron and the constant amount of delayed neutron precursor is

$$
\begin{aligned}
\frac{d n}{d t}= & -\frac{n}{l}+\frac{\left(1+k_{e x}\right)\left(1-\beta_{e f}\right)}{l} n \\
& +\frac{n_{0}}{l} \beta_{e f}\left(1+k_{e x}\right) .
\end{aligned}
$$

Assuming the cell model such that the coolant flows through the gap between fuel and moderator region, the temperature equations become

$$
\left\{\begin{array}{l}
\frac{d T_{1}}{d t}=a_{1}\left(n-n_{0}\right) \\
\frac{d T_{2}}{d t}=a_{2}\left(n-n_{0}\right),
\end{array}\right.
$$

where

\author{
$T_{1}:$ Fuel temperature \\ $T_{8}:$ Moderator temperature \\ $a_{1,2}$ : Coefficient of power removal in the \\ fuel and the moderator, respectively \\ $n_{0}:$ Initial neutron density.
}

The reactivity equation is

$$
k_{e x}=\Delta k-r_{1}\left(T_{1}-T_{1}^{0}\right)+r_{2}\left(T_{2}-T_{2}^{0}\right),
$$

where

$\Delta k$ : Excess reactivity induced from the outside cause

$r_{1}$ : Fuel temperature coefficient of reactivity, assumed as a negative number

$r_{2}$ : Moderator temperature coefficient of reactivity, assumed as a positive number.

Introducing the new variables,

$$
\begin{aligned}
& x=\frac{n-n_{0}}{n_{0}} \\
& y=\frac{T_{1}-T_{1}^{0}}{a_{1} n_{0}} \\
& z=\frac{T_{2}-T_{2}^{0}}{a_{2} n_{0}},
\end{aligned}
$$

where $T_{\mathrm{i}}^{0}$ and $T_{\mathrm{z}}^{0}$ are the steady state temperature of the fuel and moderator, respectively. Eqs. (23) and (24) become

$$
\begin{aligned}
& \frac{d x}{d t}=-\frac{x}{l}+\frac{x}{l}\left(1-\beta_{e z}\right)\left(1+k_{e x}\right)+\frac{k_{e x}}{l} \\
& \frac{d y}{d t}=x \\
& \frac{d z}{d t}=x
\end{aligned}
$$

Fortunately, Eqs.(30) and (31) have the samefunctional form and the same initial conditions:

$$
\left\{\begin{array}{l}
\frac{d y}{d t}=\frac{d z}{d t}=0 \\
y=z=0 .
\end{array} \text { at } \quad t=0\right.
$$

Therefore, Eqs. (30) and (31) can be expressed by only one equation, (30). Substituting the reactivity equation into Eq. (29) and rearranging it,

$$
\frac{d x}{d t}=A x-B x y-C y+D \text {. }
$$

The constants $A, B, C$ and $D$ can be found in Table 1. Therefore, four simultaneous equations-Eqs. (23), (24) and (25)-become only 
two simultaneous differential equations-Eqs.

(30) and (33) - to be solved.

Table 1 Coefficient of reactor kinetic equations, recycle case (Con. stant power removal model)

$$
\begin{aligned}
& A=\frac{1}{l}\left[\Delta k\left(1-\beta_{e f f}\right)-\beta_{e f f}\right] \\
& B=\frac{1}{l}\left(1-\beta_{e f f}\right)\left(r_{1} a_{1} n_{0}-r_{2} a_{2} n_{0}\right) \\
& C=\frac{1}{l}\left(r_{1} a_{1} n_{0}-r_{2} a_{2} n_{0}\right) \\
& D=\frac{\Delta k}{l}
\end{aligned}
$$

\section{Newton's Law of Cooling Model}

The application of Newton's law of cooling is a more proper and realistic representation of the heat transfer characteristics of a reactor. Eq. (24) has to be modified for this model.

$$
\begin{aligned}
& \frac{d T_{1}}{d t}=a_{1}\left(n-n_{0}\right)-b_{1}\left(T_{1}-T_{1}^{0}\right) \\
& \frac{d T_{2}}{d t}=a_{2}\left(n-n_{0}\right)-b_{2}\left(T_{2}-T_{2}^{0}\right),
\end{aligned}
$$

where

$$
\begin{aligned}
& b_{1,2}: \text { Inverse time constant of the temper- } \\
& \text { ature increase in the fuel and the } \\
& \text { moderator, respectively. }
\end{aligned}
$$

The reactor kinetic equations become

$$
\begin{aligned}
& \frac{d x}{d t}=E x-F x y+G x z-H y+K z+L \\
& \frac{d y}{d t}=x-b_{1} y \\
& \frac{d z}{d t}=x-b_{2} z .
\end{aligned}
$$

Constants $E, F, G, H, K$ and $L$ can be found in Table 2.
Table 2 Coefficient of reactor kinetic equations, recycle case (Newton's law of cooling model)

$E=\frac{1}{l}\left[\Delta k\left(1-\beta_{e f}\right)-\beta_{e f]}\right]$
$F=\frac{1}{l}\left(1-\beta_{e f}\right) r_{1} a_{1} n_{0}$
$G=\frac{1}{l}\left(1-\beta_{e f}\right) r_{2} a_{2} n_{0}$
$H=\frac{1}{l} r_{1} a_{1} n_{0}$
$K=\frac{1}{l} r_{2} a_{2} n_{0}$
$L=\frac{\Delta k}{l}$

\section{SUmmary}

Reactor kinetic equations generally applicable for a plutonium- and/or thorium-recycled! reactor were developed. It was shown that the use of the effective fraction of the delayed neutrons, $\beta_{\text {efs }}$, which was defined in Eq. (22), and the assumption of the constant amount of delayed neutron precursors made it possible to reduce the complicated reactor kinetic equations of a plutonium- and/or thorium-recycled. reactor to the simple ordinary reactor kinetic equations.

The author extends his sincere thanks to. Prof. Manson Benedict of Massachusetts Institute of Technology, not only for his everready encouragement and his invaluable advices on this work, but also for his friendship and counsel during the years of his study at the Institute. The author also wishes his thanks. to Mr. Winnett Boyd of Arthur D. Little of Canada, Ltd, for generously providing him. with the informations regarding to this work.

(Received May 28, 1962) 